\title{
Assessing the Role of Simplicity in the Continuous Use of Mobile Apps
}

\author{
Silas Formunyuy Verkijika, University of the Free State, South Africa
}

\begin{abstract}
The proliferation of smartphones has provided a huge market for mobile apps and created a massive industry for developers/designers creating various mobile apps for use in our daily activities. However, with many mobile apps competition for users attention, the continued use of these apps has become a growing concern as users easily dump one app for another even after just a single use. This study examined the role of simplicity in the continuance use of mobile apps. The study extended the ECM with simplicity and showed that simplicity had a significant direct positive influence on confirmations, perceived usefulness, and satisfaction as well as a significant total indirect effect on continuance intentions. Additionally, all the ECM proposed relationships were confirmed. The study extends the current literature on continuance use of mobile apps by demonstrating the relevance of a concrete factor that developers/designs can manipulate to improve the continuance use for their mobile apps.
\end{abstract}

\section{KEYWORDS}

Simplicity, Expectation Confirmation Model (ECM), Continuance Use, Mobile Apps

\section{INTRODUCTION}

Existing forecast suggests that there are about 2.53 billion smartphones around the globe in 2018 and the number is expected to increase by $18 \%$ in 2019 (Statista, 2018a). This enormous market of smartphone devices has been a great motivation for software developers to create a wide range of apps that can be easily downloaded and used by smartphone users. Many software developers see mobile app development as a lucrative sector given the growing use of mobile apps by users to automate various daily functions such as entertainment, communication, shopping, and productivity (Kim $\&$ Baek, 2018). This has resulted in enormous growth in mobile apps as the Google Play store, for example, saw an increase in mobile apps from just 30000 apps in March 2010 to 3.6 million apps in March 2018 (Statista, 2018b).

Despite the huge potential in the mobile app market space, a growing challenge is the continuance use of these apps, an issue that researchers have emphasized as a pressing concern that needs to be understood and addressed (Ding, 2018; Kim \& Baek, 2018). This follows from the growing evidence that about a quarter of users tend to discontinue the use of mobile apps after the first use, while about $63 \%$ use an app less than ten times (O'Connell, 2017). Also, existing statistics estimate that about $77 \%$ of daily active users discontinue using a mobile app just three days after adoption and this churn rate increases to about $90 \%$ by the end of the first month (Bonnie, 2017). In fact, the wide variety of available mobile apps for similar tasks has provided smartphone users with the opportunity to easily switch from one app to another after just a single use (O'Connell, 2017). The mobile app market grows daily and creates a hyper-competitive environment (Kim \& Baek, 2018) meanwhile only a few apps are used regularly (Furner, Racherla, \& Babb, 2016).

\section{DOI: $10.4018 /$ JOEUC.2020100102}

This article, originally published under IGI Global's copyright on October 1, 2020 will proceed with publication as an Open Access article starting on January 21, 2021 in the gold Open Access journal, Journal of Organizational and End User Computing (converted to gold Open Access January 1, 2021), and will be distributed under the terms of the Creative Commons Attribution License (http://creativecommons.org/ licenses/by/4.0/) which permits unrestricted use, distribution, and production in any medium, provided the author of the original work and 
With so many apps competing for user attention, it is not surprising that users are increasingly impatient to continue holding on to apps that do not meet their expectations (Ding, 2018). Hsu and Lin (2016) emphasize that before downloading an app, users form expectations regarding the performance of the app and thus their initial use of the app serves as the benchmark to compare their expectations to the actual performance of the app which then determines whether or not they decide to continue using the app. This view is in line with the expectation-confirmation model (ECM) which postulates that confirmation of expectations is a strong determinant of continuance intentions in the context of information systems (IS) (Bhattacherjee, 2001). In fact, many studies (e.g. Chen, Yen, \& Peng, 2018; Jia, Guo, \& Narnes, 2017; Joo, Park, \& Shin, 2017; Kim, 2010; Lin, Featherman, \& Sarker, 2017; Oghuma, Libaque-Saenz, Wong, \& Chang, 2016; Steelman \& Soror, 2017; Thong, Hong, \& Tam, 2006) on IS continuance over the years including those focusing on mobile apps (e.g. Hu \& Zhang, 2016; Tam, Santos \& Oliveira, 2018) have used the ECM as the theoretical base for understanding continuance intentions.

While these prior studies using the ECM have provided new insights by extending the model and showing its applicability in numerous contexts, only few of these studies (Baker-Eveleth \& Stone, 2015; Oghuma et al., 2016; Roca, Chiu, \& Martinez, 2006; Veeramootoo, Nunkoo, \& Dwivedi, 2018) have paid attention to factors or constructs experienced by users during their interaction with a product which shapes their confirmations of expectations regarding the product. This is particularly important as the ECM upholds the notion that confirmation is a central construct that influences perceived usefulness and satisfaction which in turn, determines continuance intentions. As such, since little attention has been paid to ex-post factors that influence the confirmations dimension, service providers might have little understanding of what to do when user's expectations are not met following their actual interactions with their systems. One of such aspects that have been pronounced by some researchers is system quality (Baker-Eveleth \& Stone, 2015; Roca, Chiu, \& Martinez, 2006). Existing evidence suggests that system quality influences users confirmations regarding a given IS system (Roca et al., 2006). This view is grounded in expectation confirmation theory (ECT). It is important to note here that Bhattacherjee (2001) developed the ECM from the ECT. According to the ECT, product performance during interaction has a direct effect on user confirmation of expectations regarding the product (Bhattacherjee, 2001; Oliver, 1990). In the context of information systems, researchers (Cheng, 2014; Koo, Wati, Park, \& Lim, 2011; Roca et al., 2006; Sharma \& Sharma, 2019) have increasingly expressed system quality as one of the vital aspects of system performance. Additionally, system quality attributes are considered as relevant positive signals that could shape user confirmations regarding a given system (Pee, Jiang \& Klein, 2018). As such, prior studies have increasingly called for a need to extend the ECM with system quality attributes (Cheng, 2014; Koo et al., 2011; Pee et al., 2018; Roca et al., 2006), especially as system quality not only confirms user expectations but also enhances their satisfaction with the system.

However, system quality is quite broad and can include aspects like usability, availability, reliability, adaptability, simplicity and response time (Bryson, 2017; DeLone \& McLean, 2003). As such, it is vital to examine specific system quality dimensions for various IS systems (e.g. BakerEveleth \& Stone, 2015). In the context of mobile apps, simplicity is considered as one of the key aspects of system quality (Fischer, 2016; Yokuş \& Yelken, 2017), yet it has been highly overlooked in research on the continuance use of mobile apps. At the time of this study, there is no apparent evidence of any study that has extended the ECM with simplicity or explicitly examined its role in IS continuance use. There are increasing calls for mobile app developers to design for simplicity (Barker, 2017; Fischer, 2016; Shaoolian, 2017; Yokuş \& Yelken, 2017). Nevertheless, there is still a dearth of research to support the importance of simplicity especially in addressing the poor level of user retention with respect to mobile apps. While designing for simplicity is considered as a best practice for mobile developers following the "KISS" principle (Banga \& Weinhold, 2014), several industry reports suggest that lack of simplicity is among the common mistakes that mobile app developers still make (Chapman, 2017; Mcconville, 2017). But, without empirical evidence, it is 
difficult to conclude that the lack of simplicity could be responsible for the high mobile app churn rates. This follows from the paradox of simplicity which suggests that even though simplicity has several benefits, users often tend to prefer complex systems (Eytam, Tractinsky, \& Lowengart, 2017). As such, although simplicity is a valuable system quality dimension, it is not clear following from the paradox of simplicity whether it actually enhances or reduces the continuance use of mobile apps. Thus, the present study intends to evaluate whether simplicity as a quality attribute can play a significant role in confirming mobile user's expectations and the subsequent appraisal of the usefulness and satisfaction with mobile apps. In answering this question, the present study will address this gap by examining the role of simplicity in the continuance use of mobile apps as the ECM posits that confirmation of expectations, satisfaction and usefulness are instrumental in the continuance use of information systems. Moreover, incorporating simplicity with the ECM is not only useful because of its potential as a quality dimension that needs to meet user expectations, but also because of its central role in user satisfaction (Choi \& Lee, 2012) and its potential to influence perceived usefulness (Banga \& Weinhold, 2014), all of which are central components of the ECM.

The rest of the paper is structured as follow. The second section presents a background of the study by reviewing the ECM and the concept of simplicity which are the two theoretical underpinnings that are combined to develop the proposed model. Afterward, the theory and development of hypotheses are presented in the third section. The fourth section presents the methodology, while the fifth section presents the data analysis which comprises the measurement and structural models. The sixth section focuses on the discussions of the findings, while the last section presents the concluding thoughts.

\section{BACKGROUND}

\section{Expectation Confirmation Model (ECM)}

The ECM was developed from the Expectation-confirmation theory (ECT) by Oliver (1980), which is a widely validated cognitive theory in the context of consumer behavior that is mostly used to explain the repurchase intentions and the continuous use of a given service by consumers. The fundamental assumption of the ECT is the view that an individual's desire to continuously engage in a given behavior is often a consequence of the satisfaction that he/she experienced during any prior engagement in the behavior as well as the extent to which their beliefs regarding the behavior were confirmed. The ECT presents a logical sequence that attempts to explain a path that consumers take to form a repurchase intention or the intention to continue using a given service. The ECT sequences commence prior to the initial purchase or adoption of a product/service offering whereby the consumers form an initial expectation about the offering. At a subsequent stage when consumers have the chance to consume the product/service offering, they also form perceptions regarding the actual performance of the system and then use these perceptions as a benchmark to compare the actual performance of the offering with the initial expectations (Halilovic \& Cicic, 2013). This comparison forms the basis for determining their level of satisfaction with the offering as consumers only become satisfied when the perceived performance meets or surpasses their expectations (Oliver, 1980). Thereafter, satisfied consumers develop a repurchase intention or the desire to continue using a given service while dissatisfied ones discontinue any further consumption of the offering (Oghuma et al., 2016; Thong et al., 2006).

The ECT was used by Bhattacherjee (2001) as the theoretical foundation to develop the ECM in order to provide an understanding of the continuance use of information systems. He likened the continuance use of information systems to the consumer's repurchase intention in the ECT which could be influenced by the level of satisfaction experienced by a user when using the system. Like the ECT, he argued that the continual use of information systems also followed a logical sequence starting with the initial acceptance of the system, then experiencing the system and lastly making ex-post decisions regarding the system (Bhattacherjee, 2001; Chen et al., 2018 Oghuma et al., 2016). The ECM (Figure 1) postulates that a user's continuance intentions regarding a given system are a direct consequence of the satisfaction experienced when using the system as well as the perceptions 
of any possible benefits that the user might gain from continuing to use the system. Moreover, these perceived benefits and level of satisfaction are influenced by the user's confirmation of their pre-use expectations about the system.

\section{Simplicity}

Simplicity is a leading principle in user interface design that has been advocated by researchers for several decades (Nielsen, 1999; Maeda, 2006). As such, human-computer interaction experts have often emphasized the need to develop simple interfaces as they are often easy to use, which is an important usability concept (Colborne, 2010). Most often, when systems are not optimally developed from a simplicity viewpoint, the complex design might seem initially attractive but ultimately the complexity decreases user satisfaction (Thompson, Hamilton, \& Rust, 2005). Also, simplicity can be vital in signaling the quality of a product and thus play a vital role in the adoption and continuance use of a system (Miyamoto, 2013). Simplicity is a multifaceted concept that comprises several dimensions. In the present study, simplicity will be viewed in terms of simplicity in visual aesthetics, reduction, and dynamic complexity.

Visual aesthetics refers to the level of attractiveness or beauty of a given interface (Moshagen \& Thielsch, 2010). Attractiveness is often a subjective issue (Eytam et al., 2017), however, the general goal when developing an interface is to ensure that the majority of the users can consider it attractive enough to use (Choi \& Lee, 2012). The visual appearance of a system plays a vital role in influencing the positive perceptions that a user has about the system. For example, many users generally perceive attractive interfaces to have good usability (Lavie \& Tractinsky, 2004; Lazard et al., 2016). Likewise, users often tend to complete tasks more successfully on attractive systems which in turn, enhance their level of satisfaction with the system (Cyr et al., 2008; Moshagen \& Thielsch, 2010).

Reduction encompasses the aspects of interface design which ensures that only essential steps are needed to complete a given task on the system (Maeda, 2006). Interfaces that are not optimally designed from a reduction perspective often tend to have intricate and unnecessary steps that a user needs to follow in order to complete a task on the system (Choi \& Lee, 2012; Lee, Moon, Kim, \& Yi, 2015). Consequently, such systems are likely to frustrate users when they encounter difficulties and subsequently diminish their satisfaction with the system. Consequently, designers are encouraged to incorporate reduction principles into their designs, especially as reduction can be applied in many parts of the interface including structural, navigational, and functional aspects (Lee et al., 2015).

Lastly, dynamic complexity refers to the ambiguity experienced by users when interacting with an interface (Choi \& Lee, 2012). An ambiguous interface basically has different possible interpretations

Figure 1. ECM model

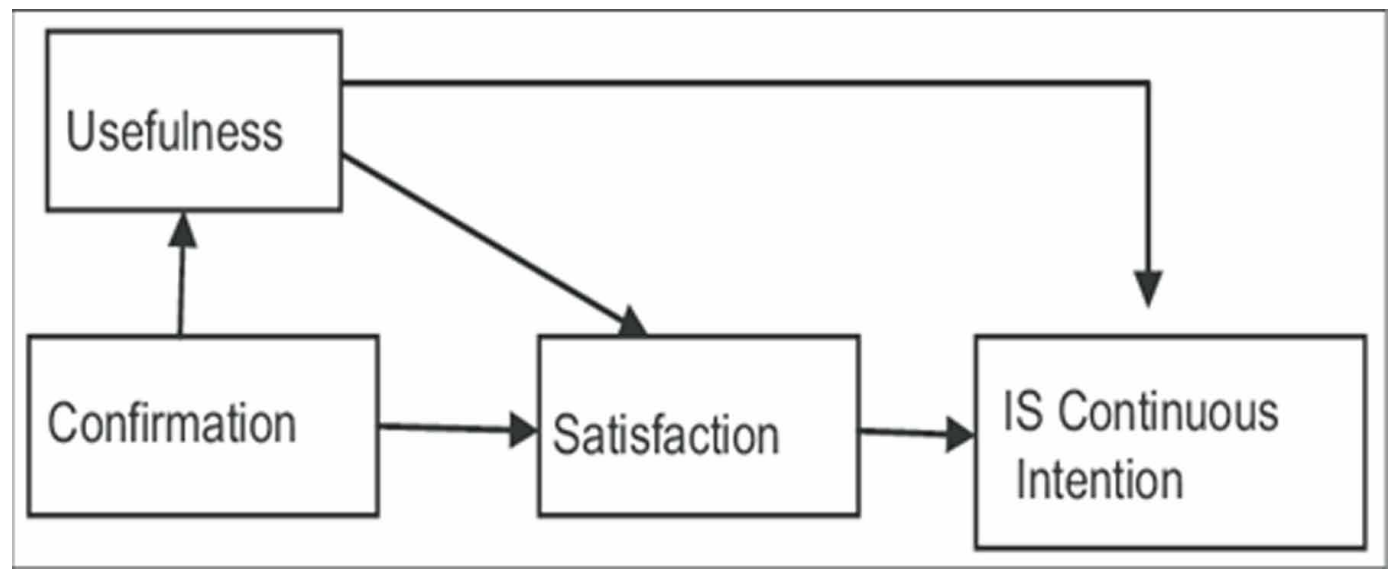


of the same information cues. As such, an interface that lacks clarity in its cause-effect chain is considered to be dynamically complex. It is important to design interfaces that are not dynamically complex as a means to enhance the overall usability of the system as user expectations are likely to be met due to the clarity of the cause-effect chain. Eliminating user interface ambiguity is likely to enhance ease of use and user satisfaction (Thimbleby, 1982).

\section{THEORY AND DEVELOPMENT OF HYPOTHESES}

\section{ECM Relationships}

The ECM hypothesizes five relationships that have been widely tested and validated. These relationships are as follows: Confirmation of expectations has a positive influence on user satisfaction (1) and perceived usefulness (2). Perceived usefulness has a direct influence on continuance intentions (3) as well as an indirect influence through its direct influence on user satisfaction (4). Lastly, user satisfaction has a direct positive influence on continuance intentions (5). Several researchers over the years who have either adopted or modified the ECM have confirmed these hypotheses in various contexts. The definition of the four constructs in the ECM is presented in Table 1.

Table 1. Definitions of ECM constructs

\begin{tabular}{|l|l|l|}
\hline Construct & Definition & Sources \\
\hline Confirmation & $\begin{array}{l}\text { The extent to which a user's actual experience with a system } \\
\text { confirms their initial expectations. }\end{array}$ & Oghuma et al. (2016) \\
\hline $\begin{array}{l}\text { Perceived } \\
\text { usefulness }\end{array}$ & $\begin{array}{l}\text { The extent to which users believe that they will benefit from } \\
\text { using a given information system. }\end{array}$ & Chen et al. (2018) \\
\hline Satisfaction & $\begin{array}{l}\text { The extent to which users are satisfied after comparing their } \\
\text { actual experience with a system to their initial expectations. }\end{array}$ & $\begin{array}{l}\text { Bhattacherjee (2001); Oliver } \\
\text { (1980) }\end{array}$ \\
\hline $\begin{array}{l}\text { Continuance } \\
\text { intentions }\end{array}$ & A user's intention to continue using a given system in the future. & $\begin{array}{l}\text { Chen et al. (2018); Oghuma } \\
\text { et al. (2016) }\end{array}$ \\
\hline
\end{tabular}

When expectations are confirmed, users tend to increase their perceptions regarding the perceived usefulness of the system. This view has been supported by several studies that applied the ECM across various contexts (Jia et al., 2017; Joo et al., 2017; Kim, 2010; Oghuma et al., 2016; Steelman \& Soror, 2017; Thong et al., 2006). Likewise, users tend to have an increased level of satisfaction with a given technology when their experience of the technology meets or surpasses their expectations, and this has been empirically supported across different context of information systems (Chen et al., 2018; Jia et al., 2017; Joo et al., 2017; Kim, 2010; Oghuma et al., 2016; Steelman \& Soror, 2017; Thong et al., 2006). Moreover, Bhattacherjee (2001) expected that the important role played by perceived usefulness in pre-adoption as captured in the technology adoption model (TAM) was likely to continue to continuance intentions following the user's perceptions regarding the ex-post usefulness of the technology. Likewise, user satisfaction with a given technology is generally higher for individuals who perceive the usefulness of the technology to be high. These views have been widely supported as several studies have shown that perceived usefulness has a direct positive influence on continuance intentions (Jia et al., 2017; Joo et al., 2017; Kim, 2010; Oghuma et al., 2016; Steelman \& Soror, 2017; Thong et al., 2006) and user satisfaction (Jia et al., 2017; Joo et al., 2017; Lin et al., 2017; Oghuma et al., 2016; Thong et al., 2006). Lastly, satisfaction is widely recognized as a vital determinant of post-adoption behavior (Bhattacherjee, 2001; Thong et al., 2006). Users who are dissatisfied with a given technology are likely to dump it and look for an alternative, thus leaving only the satisfied 
users to continue using the technology (Halilovic \& Cicic, 2013; Oghuma et al., 2016). The positive influence of satisfaction on continuance intentions have been widely validated in several contexts (Chen et al., 2018; Jia et al., 2017; Joo et al., 2017; Kim, 2010; Lin et al., 2017; Oghuma et al., 2016; Steelman \& Soror, 2017; Thong et al., 2006). Following from the above discussion, it is expected that all the relationships hypothesized by the ECM will hold true in the present study.

\section{Extending the ECM with Simplicity}

The present study conceptualizes simplicity as a second-order formative factor following from prior studies (Choi \& Lee, 2012; Lee et al., 2015). This is because each of the first-order sub-constructs of simplicity measures a specific attribute of simplicity while at the same time being part of the overall perception of simplicity. Therefore, a change in each first-order sub-construct will cause a change in the overall perception of simplicity; however, changes in one of the sub-constructs do not necessarily result in changes in other sub-constructs. In this study, the first-order sub-constructs of simplicity were limited to three dimensions (i.e. visual aesthetics, reduction, and dynamic complexity). The choice of these three sub-constructs was motivated by the context of the study. As earlier indicated, many users abandon mobile apps after a single use. As such, this study focused on user's appraisal of simplicity during the first interaction with a mobile app. Thus, it was imperative to select simplicity facets that can be adequately evaluated after the first interaction with an app. After an evaluation of the simplicity facets discussed in prior studies (Choi \& Lee, 2012; Lee et al., 2015) visual aesthetics, reduction and dynamic complexity were selected for the present study as these were believed to be factors relevant in the context of mobile apps, and that users could successfully evaluate them during their first interaction. Moreover, these three facets were also representative of all the three broad domains of simplicity proposed by Choi and Lee (2012) namely: simplicity in information design (i.e. reduction), simplicity in task complexity (i.e. dynamic complexity) and simplicity in visual aesthetics (visual aesthetics).

Simplicity can play a vital role in understanding continuance intentions as an integral part of the ECM. There are three reasons that make simplicity an important component to integrate into the ECM. Firstly, simplicity can be instrumental in providing insights as to why the user's expectations after using an information system are confirmed or not. Prior studies have shown that users are likely to have positive confirmations of a system when their expectations regarding the quality of the system are met (Roca et al., 2006). Since simplicity is a key quality attribute and at the same time a foundation for creating other aspects of system quality (Bryson, 2017), simple products would likely influence positive confirmations. For example, one expectation that users often have when using a system is the expectation of good usability as users generally expect systems to easy to use. This view was supported by Baker-Eveleth and Stone (2015) who showed that usability had a direct positive influence on user confirmation of expectations when using an e-book. It is widely acknowledged that the visual aesthetic facet of simplicity significantly enhances perceptions of systems usability (Kinnett, 2017; Pavlas, Lum \& Salas, 2010). This follows from the aesthetic-usability effect which suggests that users tend to perceive aesthetic products as more usable. Likewise, products that are optimally designed from a reduction and dynamic complexity perspective are easy to use (Choi \& Lee, 2012). As such, these facets of simplicity can play a vital role here as good usability is often a direct outcome of design simplicity, since simplicity (i.e. visual aesthetics, reduction, and dynamic complexity) results in user interfaces that are less cluttered, easy to use and easy to understand and learn how to interact with (Lee et al., 2015). Hence, the present study hypothesizes that:

\section{H1: Simplicity will have a significant positive influence on confirmation of expectations}

Secondly, simplicity has the potential to influence the ex-post perceived usefulness of a given technology. This can be achieved in several ways. For example, simplicity is the foundation for ease of use (Lee et al., 2015; Lazard et al., 2016), which is a key determinant of perceived usefulness. In line 
with the aesthetic-usability effect, Lazard et al. (2016) showed that a positive appraisal of aesthetic simplicity enhanced the perception of a system's ease of use which in turn positively influenced the perceived usefulness of the system. Likewise, since the reduction and dynamic complexity dimensions of simplicity also help to improve user perception of ease of use (Choi \& Lee, 2012; Lee, 2014), they are also likely to contribute to perceived usefulness given the positive link between ease of use and perceived usefulness (Lazard et al., 2016; Yoon, 2018). Banga and Weinhold (2014) argue that simple system designs (i.e. systems designed optimally from a reduction perspective) will always be seen as more useful to users. Additionally, a system that is optimally designed from a simplicity standpoint often tends to be perceived as having a good user-interface design quality (Choi \& Lee, 2012) which is another determinant of perceived usefulness (Garcia, 2012). This suggests that design simplicity might be instrumental in shaping users perceptions of the usefulness of the system as these users will generally see the system as easy to use and of good quality. For example, Lazard et al. (2016) showed a significant positive correlation between aesthetic simplicity and perceived usefulness. Hence the following hypothesis:

\section{H2: Simplicity will have a significant positive influence on perceived usefulness}

Thirdly, simplicity plays a vital role in enhancing the satisfaction associated with using a given technology (Choi \& Lee, 2012). Interaction with complex user interfaces is often associated with an unwarranted mental load that may tend to induce extra cognitive strain on the user which might subsequently diminish the level of satisfaction derived from the interaction (Thompson, Hamilton \& Rust 2005). So, by adopting the reduction principle in system design, user satisfaction can be improved since reduction reduces mental workload by allowing users to complete a task with few steps. Besides, researchers over the years have shown that complex products tend to cause feature fatigue, which is a key concern as feature-laden products have been known to frustrate users at the expense of user satisfaction (Goodman and Irmak, 2013; Thompson et al., 2005). In fact, users of a given technology are likely to experience greater satisfaction when their appraisal of the user interface is positive, and this is often experienced with simplified interface designs which are known for providing a satisfying user experience (Wolfinbarger \& Gilly, 2001). The visual aesthetic facet of simplicity especially plays an important role in this aspect as it generally always results in a positive appraisal of a system (Cyr et al., 2008). Similarly, systems that are designed properly from a dynamic complexity viewpoint provide predictability in the actions and expected outcomes from user interactions (Choi \& Lee, 2012). This can play a vital role in enhancing user satisfaction as predictability has been known to positively influence user satisfaction (Gajos et al., 2008). Moreover, existing evidence shows that reduction, visual aesthetics, and dynamic complexity are positively correlated with user satisfaction (Choi \& Lee, 2012; Lee et al., 2015). Furthermore, Choi and Lee (2012) showed that simplicity as a second-order formative construct (including these three facets) had a significant positive influence on user satisfaction. Hence the following hypotheses are proposed:

\section{H3: Simplicity will have a significant positive influence on satisfaction}

In Summary, the present study extended the ECM with simplicity in order to provide a deeper understanding of continuance intentions (Figure 2). In addition to the five ECM hypotheses, three hypotheses were added which focused on the role played by simplicity in influencing confirmation, perceived usefulness, and satisfaction. Another construct titled continuance use has also been added to the model as a means to capture user behavior a month's post the study to determine if they acted on their intentions. Generally, most studies based on the ECM focus only on continuance intentions (e.g. Jia et al., 2017; Kim, 2010; Oghuma et al., 2016; Steelman \& Soror, 2017; Thong et al., 2006) since intentions are considered as good predictor of actual behavior (Bhattacherjee, 2001). However, 
with mobile apps, it would be good to examine post use behavior as users sometimes discontinue use of mobile apps only after a single use or within three days from adoption as earlier discussed. Hence the following hypothesis is proposed:

\section{H4: Continuance intentions will have a significant positive influence on continuance use}

The proposed model is presented in Figure 2. Simplicity is conceptualized as a second-order formative factor composed of three sub-constructs (i.e. aesthetic simplicity, dynamic complexity, and reduction). The hypothesized associations are indicated by $\mathrm{H} 1$ to $\mathrm{H} 4$. The positive sign (+) indicates the expected relationship that has been widely validated in the ECM.

Figure 2. Proposed model

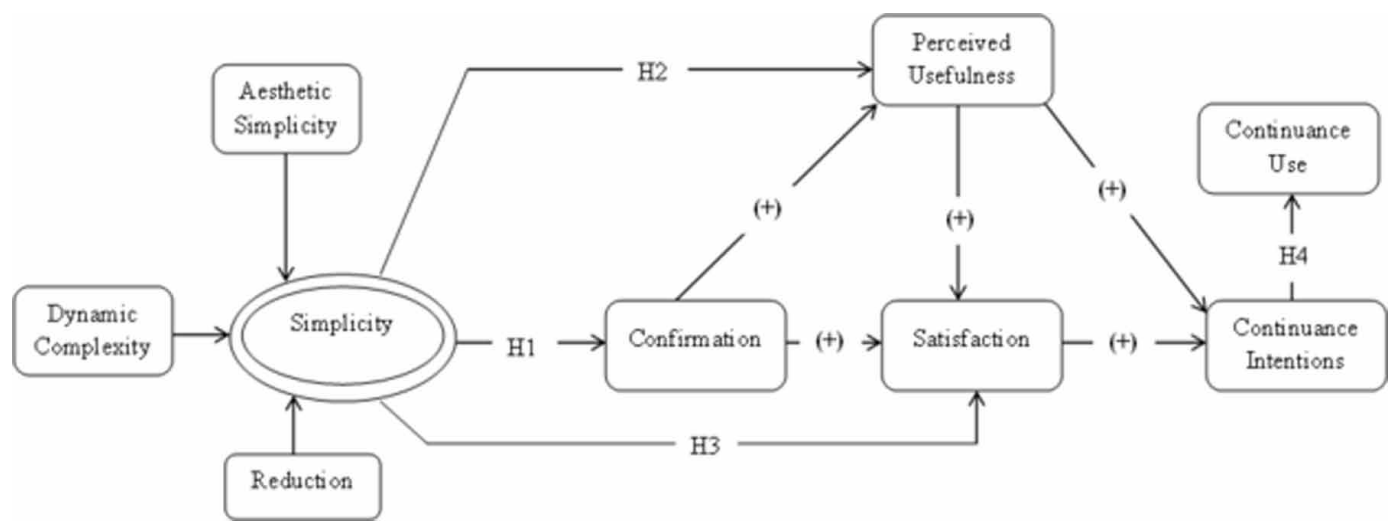

\section{METHODOLOGY}

The present study adopted a single-group post-test only design to evaluate the proposed model. With this design, a single-group of participants is allowed to interact with a given product/system and then report on their post-use experiences (Jackson, 2012). This design was adopted in the present study because all the participants interacted with the selected mobile app for the first time. Growing evidence suggests that many mobile app users make continuance decisions after a single use of the mobile app and some immediately decide to uninstall mobile apps after a single use (Bonnie, 2017; O'Connell, 2017). As such, the first post-use experiences of the users can be instrumental in determining whether or not they intend to continue using the app. Since the selected sample of the study was students, the study used a student time management app as the selected mobile app as students are the target users of the app. Seeing as all the participants were using the mobile app for the first time, it was important to carefully select participants who are firstly interested in adopting the app and who have not used it before. As such the selection of participants followed four steps as follows: (1) an invitation was made to potential participants. The invitation was accompanied by an information sheet that explained the purpose of the study and the requirements from the participant; (2) A total of 108 students responded to the invitation; (3) All the students who responded to the invitation were shown information about the app as presented in the app store for them to decide if it's an app they will adopt by downloading to test and use; (4) From these 108 students, 3 were excluded from the study because they were already using the app and so their first time experience could not be evaluated. Five others were also 
excluded because their initial decision after viewing the app was not to download and try it. As such, their continuance intentions could not be evaluated given that their initial decision was not to use the app. The remaining 100 students made the decision to download and use the app after evaluating the information about the app presented on the app store and thus were selected for the study since their continuance intentions regarding app could be evaluated.

This sample size of 100 students was deemed sufficient for the present study after the following considerations. Firstly, taking simplicity as a second-order formative factor that comprises of three sub-constructs (i.e. aesthetic simplicity, reduction, and dynamic complexity) an evaluation of the model based on Daniel Soper's A-priori sample size calculator for structural equation models suggest that the recommended minimum sample size is 92 . Secondly, power analysis using the G*Power free software recommended by Hair et al (2017) for structural equation modeling suggested a minimum sample size of 89. Lastly, since the study used partial least square (PLS) structural equation modeling (SEM) which is known for providing accurate results even for small samples sizes, the sample of 100 was also sufficient when applying the "10-times rule" that is popularly used as a guide for selecting a sample size for PLS-SEM analysis (Hair et al., 2017).

Each of the students was given 15 minutes to interact with the app following a set of the guided tasks which were carefully designed by the researcher to ensure that all key functions of the app were evaluated. After the evaluation, the participants were then provided with a post-test questionnaire to evaluate their experiences based on the simplicity of the design and the ECM constructs. The measures for simplicity were adapted from Choi and Lee (2012), while those for the ECM constructs were adapted from prior studies (Bhattacherjee, 2001; Jia et al., 2017; Joo et al., 2017; Oghuma et al., 2016; Steelman \& Soror, 2017; Thong et al., 2006). One month after the evaluations, the participants were contacted to see those that had continued to use the app. This timeframe was selected as churn rates for mobile apps often reach $90 \%$ within the first month (Bonnie, 2017). This provided the measure for the continued used construct which as captured as a dummy variable with " 1 " representing those who continued using the app and " 0 " otherwise.

The respondents were $56 \%$ male and $44 \%$ female. Also, the majority of the respondents were below the age of 25 years (86\%). All the participants owned a smartphone and many of them (89\%) had used smartphones for more than a year. Android was the dominant smartphone operating system as $97 \%$ of the participants used android phones. After the one month post-test period, it was seen that $21 \%$ of the participants had continued to use the mobile app.

\section{DATA ANALYSIS}

PLS-SEM was conducted in this study following a two-stage analysis process which included the measurement model and the structural model. The SmartPLS software version 3.0 was used for the data analysis.

\section{Measurement Model}

The measurement, model was used to assess the reliability and validity of the constructs. Cronbach's alpha and composite reliability were used to assess the internal consistency reliability. Cronbach alpha values ranged from 0.752 to 0.951 while the composite reliability values ranged from 0.890 to 0.976 . All these values are above the commonly used threshold of 0.7 thus confirming the internal consistency reliability of the constructs (Hair, Hult, Ringle \& Sarstedt, 2017). The average variance extracted (AVE) was used to assess the convergent validity of the constructs following the recommended threshold of 0.5. All the observed AVE values were far above 0.5 with the lowest AVE value being 0.772. As such, the constructs are considered to demonstrate an acceptable level of convergent validity (Table 2).

Discriminant validity was assessed using the Heterotrait-Monotrait Ratio (HTMT) following the arguments that the commonly used Fornell-Larcker criterion and cross-loadings might not reliably detect discriminant validity issues (Hair et al., 2017). Hair et al. (2018) suggest using 0.85 as the 
Table 2. Reliability and convergent validity

\begin{tabular}{|c|c|c|c|}
\hline Construct & Cronbach's alpha & $\begin{array}{c}\text { Composite } \\
\text { reliability }\end{array}$ & $\begin{array}{c}\text { Average variance } \\
\text { extracted (AVE) }\end{array}$ \\
\hline Aesthetic Simplicity (AS) & 0.877 & 0.924 & 0.803 \\
\hline Confirmation (CE) & 0.867 & 0.918 & 0.790 \\
\hline Continuance intentions (CI) & 0.951 & 0.976 & 0.953 \\
\hline Dynamic Complexity (DC) & 0.752 & 0.890 & 0.801 \\
\hline Perceived usefulness (PU) & 0.902 & 0.939 & 0.772 \\
\hline Reduction (RE) & 0.850 & 0.910 & 0.838 \\
\hline Satisfaction (SA) & 0.935 & 0.954 & \\
\hline
\end{tabular}

Continuance use $(\mathrm{CU})$ is not included in the table because it was measured as a dummy variable

threshold for measuring discriminant validity, whereby HTMT values below 0.85 will be considered as demonstrating acceptable levels of discriminant validity. From Table 3, it is observed that the HTMT values ranged from 0.149 to 0.779 , thus suggesting that there are no issues of discriminant validity.

\section{Structural Model}

Figure 3 summarizes the results of the structural equation model obtained using the SmartPLS software. The hypothesized associations were evaluated using bootstrapping with 5000 subsamples. In the model, simplicity explained $41.2 \%$ variance in confirmation of expectations while simplicity and confirmation of expectations explained $52.5 \%$ variance in perceived usefulness. The model also explained $67.4 \%$ variance in satisfaction, $57.8 \%$ variance in continuance intentions and $34.6 \%$ variance in continuance use.

The conceptualization of simplicity as a second-order construct as proposed in prior studies (Choi $\&$ Lee, 2012; Lee et al., 2015) was also validated in the present study as the sub-dimensions of aesthetic simplicity $(\beta=0.454, \mathrm{p}<0.01)$ reduction $(\beta=0.467, \mathrm{p}<0.01)$ and dynamic complexity $(\beta=0.327$, $\mathrm{p}<0.01$ ) were all positive and significantly associated with the higher-order construct (i.e. simplicity). With regards to relationships proposed by the ECM, it is observed that all the ECM relationships were supported. Confirmation had a significant positive influence on perceived usefulness $(\beta=0.237$, $\mathrm{p}<0.01)$ and satisfaction $(\beta=0.287, \mathrm{p}<0.01)$. Likewise, perceived usefulness had a significant positive influence on satisfaction $(\beta=0.204, \mathrm{p}<0.01)$ and continuance intention $(\beta=0.481, \mathrm{p}<$ $0.01)$, while the positive influence of satisfaction on continuance intentions was also significant $(\beta=$ $0.339, \mathrm{p}<0.01)$. With regards to simplicity, it is observed that simplicity has a significant positive

Table 3. Discriminant validity with HTMT

\begin{tabular}{|c|c|c|c|c|c|c|c|}
\hline & AS & CE & CI & CU & DC & PU & RE \\
\hline CE & 0.598 & & & & & & \\
\hline CI & 0.547 & 0.650 & & & & & \\
\hline CU & 0.217 & 0.323 & 0.603 & & & & \\
\hline DC & 0.530 & 0.599 & 0.690 & 0.333 & & & \\
\hline PU & 0.589 & 0.659 & 0.779 & 0.413 & 0.778 & & \\
\hline RE & 0.490 & 0.528 & 0.460 & 0.149 & 0.544 & 0.563 & \\
\hline SA & 0.538 & 0.758 & 0.714 & 0.359 & 0.724 & 0.768 & 0.697 \\
\hline
\end{tabular}




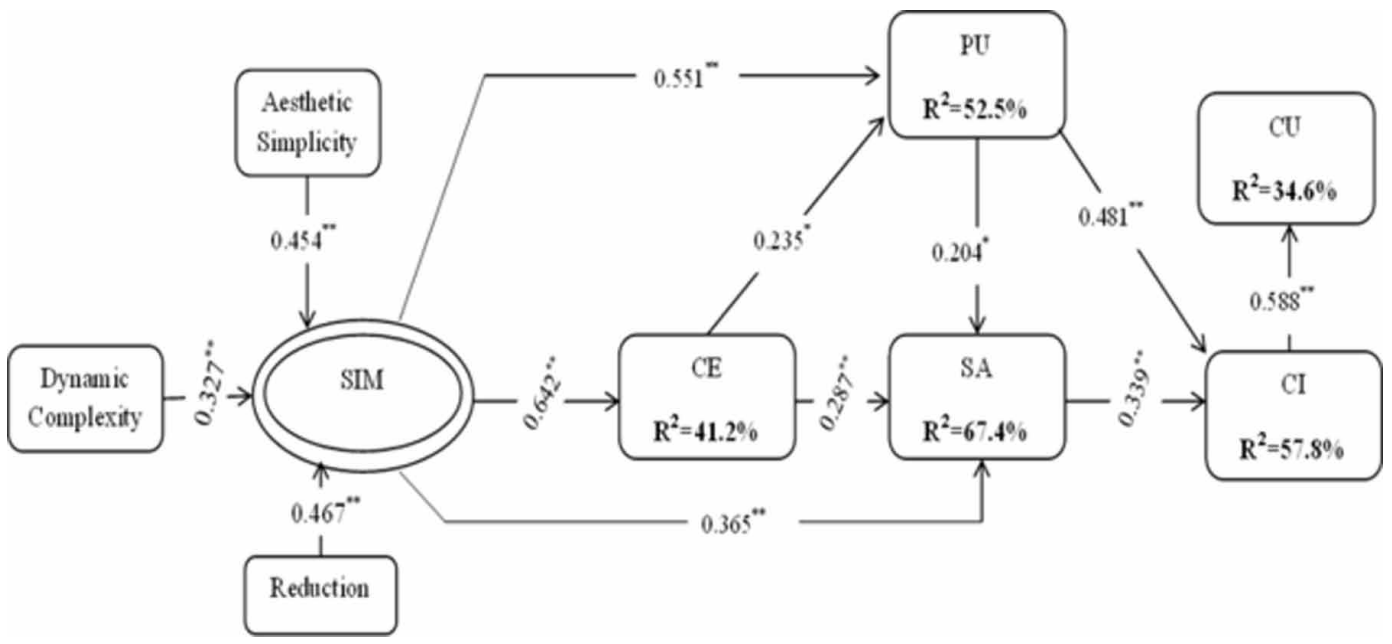

influence on confirmation of expectations $(\beta=0.642, \mathrm{p}<0.01)$, perceived usefulness $(\beta=0.551$, $\mathrm{p}<0.01)$ and satisfaction $(\beta=0.365, \mathrm{p}<0.01)$. The structural model showed support for all the hypothesized associations (Table 4). Above and beyond the hypothesized associations, it was also observed that the total indirect effect on simplicity on continuance intentions $(\beta=0.591, \mathrm{p}<0.01)$ and continuance use $(\beta=0.347, \mathrm{p}<0.01)$ was positive and significant at $1 \%$.

\section{DISCUSSIONS}

The present study extended the ECM with simplicity and found support for both the ECM and its extended version. The ECM has been widely supported by prior studies even though not all hypothesized associations of the ECM are always valid in all contexts (Chen et al., 2018; Kim, 2010). Nevertheless, in the present study, all the ECM relationships were supported which is in line with several prior studies that have also supported all the associations of the ECM (Jia et al., 2017; Joo et al., 2017; Lin et al., 2017; Oghuma et al., 2016; Steelman \& Soror, 2017; Tam et al., 2018; Thong et al., 2006). Additionally, it was observed that simplicity had a positive and significant influence on confirmation of expectations (H1), perceived usefulness (H2) and satisfaction (H3). The findings support the evidence from prior studies that have established a significant positive influence of simplicity on satisfaction (Choi \& Lee, 2012).

Table 4. Outcomes of hypotheses

\begin{tabular}{|c|c|c|c|c|c|}
\hline Hypothesis & $\begin{array}{c}\text { Constructs' } \\
\text { Relationship }\end{array}$ & $\begin{array}{c}\text { Standardized } \\
\text { path coefficient }\end{array}$ & Critical ratio & Significance (p) & $\begin{array}{c}\text { Hypothesis } \\
\text { Supported (Yes } \\
\text { / No) }\end{array}$ \\
\hline $\mathrm{H} 1$ & $\mathrm{SIM} \rightarrow \mathrm{CE}$ & $0.642^{* *}$ & 10.231 & $\mathrm{p}=0.000$ & Yes \\
\hline $\mathrm{H} 2$ & $\mathrm{SIM} \rightarrow \mathrm{PU}$ & $0.551^{* *}$ & 5.571 & $\mathrm{p}=0.000$ & Yes \\
\hline $\mathrm{H} 3$ & $\mathrm{SIM} \rightarrow \mathrm{SA}$ & $0.356^{* *}$ & 4.136 & $\mathrm{p}=0.000$ & Yes \\
\hline $\mathrm{H} 4$ & $\mathrm{CI} \rightarrow \mathrm{CU}$ & $0.588^{* *}$ & 12.263 & $\mathrm{p}=0.000$ & Yes \\
\hline
\end{tabular}

Note: ${ }^{* *} p<0.01 ;{ }^{*} p<0.05$ 


\section{Implications of the Study}

The present study makes a valuable contribution to both research and practice. For researchers, the study makes a theoretical contribution to the ongoing research on the continuance use of information systems. The ECM has been widely validated by many researchers to be a valuable model for studying continuance use of information system with several prior studies demonstrating the need for extending the model in different context of use (Jia et al., 2017; Oghuma et al., 2016; Steelman \& Soror, 2017; Tam et al., 2018; Thong et al., 2006). Confirmation of expectations is a vital component of the ECM as it directly influences post use behaviors (Bhattacherjee, 2001; Chen et al., 2018 Oghuma et al., 2016) that in turn determine whether or not a user will continue using a given information system. For systems like mobile apps, disconfirmation might require the developers of the system to go back to the drawing board and redesign such systems. However, prior research had paid little attention to ex-post factors that could influence the user's confirmation of their expectations. The present study addressed this issue by showing that simplicity plays a significant role in influencing the user's confirmation of expectations with respect to mobile apps (accounting for $41.2 \%$ ). This provides room for future studies to extend the present model with other ex-post factors that can further explain user confirmation of expectations. Additionally, simplicity also proved to be a valuable addition to the ECM as it also had a significant direct influence on perceived usefulness and satisfaction with an overall significant indirect influence on continuance intentions and use. In fact, the influence of simplicity on perceived usefulness and satisfaction was stronger than that of the original associations proposed in the ECM. Moreover, the extension of the ECM with simplicity accounted for $52.5 \%$ variance in perceived usefulness and $67.4 \%$ variance in satisfaction which is greater than what several other prior extensions of the ECM have achieved for perceived usefulness (e.g. Jai et al., 2017; Kim, 2010; Lin et al., 2017; Steelman \& Soror, 2017; Thong et al., 2006) and satisfaction (e.g. Jai et al., 2017; Kim, 2010; Lin et al., 2017; Oghuma et al., 2016; Steelman \& Soror, 2017). As such, simplicity can be seen to be a valuable construct for extending the ECM thus adding to the growing literature of continuance use of information systems.

For practitioners, the present study provides a concrete factor (i.e. simplicity) that mobile app providers and designers can manipulate to enhance the continuous use of their applications by users. The ECM has widely shown the need to develop systems that are likely to have positive confirmations and also find ways to enhance the perceived usefulness and satisfaction of the systems as these together significantly affects an individual's continuance intentions with an information system. The present study showed that simplicity, a design attribute that can be manipulated by developers and designers plays a vital role in enhancing the probability of positive confirmations as well as the user's perceptions of usefulness and satisfaction of a mobile app. These findings further reinforce the existing calls for mobile app developers and designers to design for simplicity (Banga \& Weinhold, 2014; Yokus \& Yelken, 2017). Practitioners are therefore called upon to follow the "simplicity principle" when designing mobile apps as this will not only signal product value as suggested by Miyamoto (2013) but also play a momentous role in determining the overall decision of a user to continue using the app, possibly amidst the existing alternatives. Additionally, since evidence suggests that users often tend to uninstall some mobile apps only after a single use, it would be important for providers of apps with noticeable user uninstallations to conduct a user evaluation of the simplicity of their apps and address any identified issues. Likewise, mobile app developers should encourage users to comment on the simplicity of their app so that the necessary steps can be taken to enhance the simplicity of their designs. Similar information can also be obtained from the user comments in the app store and providers of mobile apps should endeavor to quickly address any concerns about the app's Simplicity.

\section{Limitations and Avenues for Future Studies}

The limitations of the study are twofold. First, the study focused only on one mobile app (i.e. a student time management app). Even though the participants in the study were the representative users of this mobile app, it is not certain if the results can be replicated for mobile apps from other 
categories. As such, in order to generalize the important role played by simplicity in the continuance use of mobile apps, it would be imperative for future studies to test the hypothesized association in this study with different types of mobile apps. Secondly, the present study only used three facets of simplicity; however, simplicity has more than three facets used differently by different studies (Choi \& Lee, 2012; Lee et al, 2015). As such, future studies can test different facets of simplicity especially taking into account the type of mobile app that is tested and how that particular facet of simplicity can be important in the context.

Additionally, the present study showed the positive role of simplicity after the first interaction with a mobile app. However, it is uncertain whether simplicity continues to play an important role in the long run after the users are already familiar with all aspects of the mobile app. Future studies can consider a longitudinal study whereby simplicity is measured at various timeframes in order to ascertain the long term effect of simplicity on the continuance use of mobile apps. For example, does the effect of simplicity on perceived usefulness, confirmation of expectations and satisfaction diminish, increase or remain constant over time.

Additionally, while prior studies generally acknowledge simplicity as a second-order formative factor (Choi \& Lee, 2012; Lee et al., 2015), the lack of consensus on what simplicity entails is an avenue that requires careful consideration in the literature in order to provide guidance for future empirical studies on simplicity. For example, Choi and Lee (2012) conceptualized simplicity as comprising of three broad themes namely (1) simplicity in information design, (2) simplicity in task complexity and (3) simplicity in visual aesthetics. However, what Lee et al. (2015) regard as simplicity as a whole is what according to Choi and Lee (2012) refers only to one dimension of simplicity (i.e. simplicity in information design) even though both studies develop their simplicity concept from Maeda's (2006) book on simplicity. These authors acknowledge that there are limited empirical studies validating the concept of simplicity and most practitioners provide varying subjective views of what constitutes simplicity (Lee et al., 2015). Future studies need to consolidate information from these existing studies and try to provide a clear direction for researchers on how to conceptualize and empirically apply the concept of simplicity as there is growing empirical evidence that simplicity plays an important role in the use of information systems. Lastly, besides trying to provide a universal conceptualization of simplicity, it is also important for the impact of different facets of simplicity to be applied to various technologies especially as the paradox of simplicity suggests that benefits of simplicity can at times be overlooked as some users often tend to prefer complex systems (Eytam et al., 2017).

\section{CONCLUSION}

This study contributes to the growing literature that extends the ECM to better understand the continuance use of information systems with a specific focus on mobile apps. In addition to reiterating the vital role of the ECM in continuance intentions, the study further showed that simplicity was a valuable extension to the ECM as simplicity enhanced the likelihood of positive confirmations and also positively influenced perceived usefulness and satisfaction. Overall the findings of the present study provide new insights on the continuance use of mobile apps and show that developers and designers of mobile apps could possibly manipulate a concrete factor like simplicity to enhance the probability of continuance use of their mobile apps. 


\section{REFERENCES}

Banga, C., \& Weinhold, J. (2014). Essential Mobile Interaction Design: Perfecting Interface Design in Mobile Apps. Upper Saddle River, NJ: Addison-Wesley.

Barker, J. (2017). When Designing Apps, Simplicity Is Where It's At. Worryfreelabs. Retrieved from https:// www.worryfreelabs.com/designing-apps-simplicity/

Bhattacherjee, A. (2001). Understanding Information Systems Continuance: An Expectation-Confirmation Model. Management Information Systems Quarterly, 25(3), 351-370. doi:10.2307/3250921

Bryson, B. (2017). Driving Architectural Simplicity - The Value, Challenge, and Practice of Simple Solutions. Infoq. Retrieved from https://www.infoq.com/articles/driving-architectural-simplicity

Chapman, C. (2017). Mobile app design best practices and mistakes. Toptal. Retrieved from https://www.toptal. com/designers/mobile/mobile-app-design-mistakes

Chen, S., Yen, D. C., \& Peng, S. (2018). Assessing the impact of determinants in e-magazines acceptance: An empirical study. Computer Standards \& Interfaces, 57, 49-58. doi:10.1016/j.csi.2017.11.004

Cheng, Y. (2014). Extending the expectation-confirmation model with quality and flow to explore nurses' continued blended e-learning intention. Information Technology \& People, 27(3), 230-258. doi:10.1108/ITP01-2013-0024

Choi, J. H., \& Lee, H. (2012). Facets of simplicity for the smartphone interface: A structural model. International Journal of Human-Computer Studies, 70(2), 129-142. doi:10.1016/j.ijhcs.2011.09.002

Colborne, G. (2010). Simple and Usable Web, Mobile, and Interaction Design. Berkeley, CA: New Riders.

DeLone, W. H., \& McLean, E. R. (2003). The DeLone and McLean model of information systems success: A tenyear update. Journal of Management Information Systems, 19(4), 9-30. doi:10.1080/07421222.2003.11045748

Ding, Y. (2018). Modelling continued use of information systems from a forward-looking perspective: Antecedents and consequences of hope and anticipated regret. Information \& Management, 55(4), 461-471. doi:10.1016/j. im.2017.11.001

Eytam, E., Tractinsky, N., \& Lowengart, O. (2017). The paradox of simplicity: Effects of role on the preference and choice of product visual simplicity level. International Journal of Human-Computer Studies, 105, 43-55. doi:10.1016/j.jhcs.2017.04.001

Fischer, M. (2016). Why Simplicity Matters for Mobile App Developers. Dogtown Media. Retrieved from https:// www.dogtownmedia.com/why-simplicity-matters-for-mobile-app-developers/

Furner, C. P., Racherla, P., \& Babb, J. S. (2016). What we know and do not know about mobile app usage and stickiness: A research agenda. Journal International Journal of E-Services and Mobile Applications, 7(3), 48-69. doi:10.4018/IJESMA.2015070104

Gajos, K. Z., Everitt, K., Tan, D. S., Czerwinski, M., \& Weld, D. S. (2008). Predictability and accuracy in adaptive user interfaces. In Proceeding of the Twenty-sixth Annual SIGCHI Conference on Human Factors in Computing Systems (pp. 1271-1274). Academic Press. doi:10.1145/1357054.1357252

Garcia, Y. (2012). The effects of Information Systems quality on Nurses' Acceptance of the Electronic Learning System. The Journal of Nursing Research, 20(1), 19-30. doi:10.1097/JNR.0b013e31824777aa PMID:22333963

Goodman, J. K., \& Irmak, C. (2013). Having Versus Consuming: Failure to Estimate Usage Frequency Makes Consumers Prefer Multifeature Products. JMR, Journal of Marketing Research, 50(1), 44-54. doi:10.1509/ jmr.10.0396

Hair, J. F., Hult, G. T. M., Ringle, C. M., \& Sarstedt, M. (2017). A Primer on Partial Least Squares Structural Equation Modeling (2nd ed.). Thousand Oaks, CA: Sage.

Halilovic, S., \& Cicic, M. (2013). Understanding determinants of information systems users' behaviour: A comparison of two models in the context of integrated accounting and budgeting software. Behaviour \& Information Technology, 32(12), 1280-1291. doi:10.1080/0144929X.2012.708784 
Hsu, C., \& Lin, J. C. (2016). Effect of Perceived Value and Social Influences on Mobile App Stickiness and In-App Purchase Intention. Technological Forecasting and Social Change, 108, 42-53. doi:10.1016/j. techfore.2016.04.012

Hu, J., \& Zhang, Y. (2016). Understanding Chinese Undergraduates' Continuance Intention to Use Mobile BookReading Apps: An Integrated Model and Empirical Study. International Journal of Libraries and Information Studies, 66(2), 85-112.

Jia, Q., Guo, Y., \& Barnes, S. J. (2017). Enterprise 2.0 Post-adoption: Extending the Information System Continuance Model Based on the Technology-Organization-Environment Framework. Computers in Human Behavior, 67, 95-105. doi:10.1016/j.chb.2016.10.022

Joo, Y. J., Park, S., \& Shin, E. K. (2017). Students' Expectation, Satisfaction, and Continuance Intention to Use Digital Textbooks. Computers in Human Behavior, 69, 83-90. doi:10.1016/j.chb.2016.12.025

Kim, B. (2010). An empirical investigation of mobile data service continuance: Incorporating the theory of planned behavior into the expectation-confirmation model. Expert Systems with Applications, 37(10), 7033-7039. doi:10.1016/j.eswa.2010.03.015

Kim, S., \& Baek, T. H. (2018). Examining the antecedents and consequences of mobile app engagement. Telematics and Informatics, 35(1), 148-158. doi:10.1016/j.tele.2017.10.008

Kinnett, S. J. (2017). How to win at CRM: strategy, implementation, management. Boca Raton, FL: CRC Press.

Koo, C., Wati, Y., Park, K., \& Lim, M. K. (2011). Website Quality, Expectation, Confirmation, and End User Satisfaction: The Knowledge-Intensive Website of the Korean National Cancer Information Center. Journal of Medical Internet Research, 13(4), e81. doi:10.2196/jmir.1574 PMID:22047810

Lavie, T., \& Tractinsky, N. (2004). Assessing dimensions of perceived visual aesthetics of web sites. International Journal of Human-Computer Studies, 60(3), 269-298. doi:10.1016/j.ijhcs.2003.09.002

Lazard, A. J., Watkins, I., Mackert, M. S., Xie, B., Stephens, K. K., \& Shalev, H. (2016). Design Simplicity Influences Patient Portal Use: The Role of Aesthetic Evaluations for Technology Acceptance. Journal of the American Medical Informatics Association, 23(e1), e157-e161. doi:10.1093/jamia/ocv174 PMID:26635314

Lee, D., Moon, J., Kim, Y. J., \& Yi, M. Y. (2015). Antecedents and Consequences of Mobile Phone Usability: Linking Simplicity and Interactivity to Satisfaction, Trust, and Brand Loyalty. Information \& Management, 52(3), 295-304. doi:10.1016/j.im.2014.12.001

Lin, X., Featherman, M., \& Sarker, S. (2017). Understanding Factors Affecting Users' Social Networking Site Continuance: A Gender Difference Perspective. Information \& Management, 54(3), 383-395. doi:10.1016/j. im.2016.09.004

Maeda, J. (2006). The Laws of Simplicity. MA: MIT Press.

Mcconville, D. (2017). The 5 most common mistakes when it comes to mobile app design. SeamGen. Retrieved from https://www.seamgen.com/blog/mobile-app-design/

Miyamoto, S. (2013). The Signaling Value of Product Simplicity. Washington University, St. Louis, MI.

Moshagen, M., \& Thielsch, M. T. (2010). Facets of visual aesthetics. International Journal of Human-Computer Studies, 68(10), 689-709. doi:10.1016/j.ijhcs.2010.05.006

Nielsen, J. (1999). Designing Web Usability: The Practice of Simplicity (1st ed.). Berkeley, CA: Peachpit Press.

O'Connell, C. (2017). 24\% of Users Abandon an App After One Use. Localytics. Retrieved from http://info. localytics.com/blog/24-of-users-abandon-an-app-after-one-use

Oghuma, P. A., Libaque-Saenz, C. F., Wong, S. F., \& Chang, Y. (2016). An expectation-confirmation model of continuance intention to use mobile instant messaging. Telematics and Informatics, 33(1), 34-47. doi:10.1016/j. tele.2015.05.006

Oliver, R. L. (1980). A cognitive model of the antecedents and consequences of satisfaction decisions. JMR, Journal of Marketing Research, 17(4), 460-469. doi:10.1177/002224378001700405 
Pavlas, D., Lum, H., \& Salas, E. (2010). The influence of aesthetic and usability web design elements on viewing patterns and user response: An eye-tracking study. Proceedings of the Human Factors and Ergonomics Society Annual Meeting, 54(6), 1244-1248. doi:10.1177/154193121005401602

Pee, L. G., Jiang, J., \& Klein, G. (2018). Signaling effect of website usability on repurchase intention. International Journal of Information Management, 39, 228-241. doi:10.1016/j.ijinfomgt.2017.12.010

Roca, J. C., Chiu, C., \& Martinez, F. J. (2006). Understanding e-learning continuance intention: An extension of the Technology Acceptance Model. International Journal of Human-Computer Studies, 64(8), 683-696. doi:10.1016/j.jijhcs.2006.01.003

Shaoolian, G. (2017). 5 Mobile App Features Your Customers Want. Forbes. Retrieved from https://www.forbes. com/sites/gabrielshaoolian/2017/06/07/5-mobile-app-features-your-customers-want/\#54d2a9047bf9

Sharma, S. K., \& Sharma, M. (2019). Examining the role of trust and quality dimensions in the actual usage of mobile banking services: An empirical investigation. International Journal of Information Management, 44, 65-75. doi:10.1016/j.ijinfomgt.2018.09.013

Statista. (2018a). Number of Smartphone Users Worldwide from 2014 to 2020 (in billions). Retrieved from https://www.statista.com/statistics/330695/number-of-smartphone-users-worldwide/

Statista. (2018b). Number of Available Applications in the Google Play Store from December 2009 to June 2018. Retrieved from: https://www.statista.com/statistics/266210/number-of-available-applications-in-thegoogle-play-store/

Steelman, Z. R., \& Soror, A. A. (2017). Why Do You Keep Doing That? The Biasing Effects Of Mental States On IT Continued Usage Intentions. Computers in Human Behavior, 73, 209-223. doi:10.1016/j.chb.2017.03.027

Tam, C., Santos, D., \& Oliveira, T. (2018). Exploring the Influential Factors of Continuance Intention to Use Mobile Apps: Extending the Expectation Confirmation Model. Information Systems Frontiers. doi:10.1007/ s10796-018-9864-5

Thimbleby, H. (1982). Character Level Ambiguity: Consequences for User Interface Design. International Journal of Man-Machine Studies, 16(2), 211-225. doi:10.1016/S0020-7373(82)80057-6

Thompson, D. V., Hamilton, R. W., \& Rust, R. T. (2005). Feature Fatigue When Product Capabilities Become Too Much of a Good Thing. JMR, Journal of Marketing Research, 42(4), 431-442. doi:10.1509/jmkr.2005.42.4.431

Wolfinbarger, N., \& Gilly, M. C. (2001). Shopping Online For Freedom, Control, and Fun. California Management Review, 43(2), 34-55. doi:10.2307/41166074

Yokuş, G., \& Yelken, T. Y. (2017). The Adoption of Mobile Devices as Digital Tools for Seamless Learning. In S. Şad \& M. Ebner (Eds.), Digital Tools for Seamless Learning (pp. 297-324). Hershey, PA: IGI Global. doi:10.4018/978-1-5225-1692-7.ch015

Yoon, C. (2018). Extending the TAM for Green IT: A normative perspective. Computers in Human Behavior, 83, 129-139. doi:10.1016/j.chb.2018.01.032 
Silas Formunyuy Verkijika is a research fellow in the Department of Computer Science and Informatics at the University of the Free State. He holds an MSc and a PhD in Computer Information systems. His major research interest includes e-government, e-commerce, human-computer interaction, ICT4D, and information security. Some of his work has appeared in prominent journals such as Computers \& Education, the International Journal of Information management, Electronic Commerce Research and Applications, Telematics and Informatics and Computers \& Security. 